\title{
Partial nephrectomy in horseshoe kidney: Primary carcinoid tumor
}

\author{
Kamil Gokhan Seker ${ }^{1}$, Emre Sam ${ }^{1}$, Selcuk Sahin ${ }^{1}$, Mustafa Gürkan Yenice ${ }^{1}$, Ayse Gul Aktas ${ }^{2}$, \\ Abdulmuttalip Simsek ${ }^{1}$, Volkan Tugcu ${ }^{1}$ \\ ${ }^{1}$ Department of Urology, Bakırkoy Dr.Sadi Konuk Training and Research Hospital, Istanbul, Turkey; \\ ${ }^{2}$ Department of Pathology, Bakırkoy Dr.Sadi Konuk Training and Research Hospital, Istanbul, Turkey.
}

\begin{abstract}
Summary Primary neuroendocrine carcinoma of the kidney is a rarely observed clinical condition because neuroendocrine cells are not found in kidney parenchyma. It's not clinically and radiologically possible to distinguish from other kidney tumors. Incidence with horseshoe kidney anomaly, it should be considered as a definitive diagnosis for the patients with this condition. In this case report, we reported about a carcinoid tumor in horseshoe kidney in a 37-year-old woman.
\end{abstract}

KEY WORDS: Carcinoid tumor; Neuroendocrine tumor; Horseshoe kidney; Partial nephrectomy.

Submitted 8 June 2017; Accepted 4 August 2017

\section{INTRODUCTION}

Carcinoid tumors are low-grade tumors, exhibiting differentiation from neuroendocrine cells, with malignancy potential. Neuroendocrine cells in the body are also known are enterochromoffin cells or amine precursor uptake and decarboxylation cells (APUD) and they present in glandulous endocrine organs (like hypophysis, parathyroid, adrenal medulla). Neuroendocrine tumors (NETs) are most commonly present at gastrointestinal system by $73.7 \%$ and respiratory system by $25.1 \%$ where they rarely present in ovary, testis, cervix, breast, biliary tract and gall bladder $(1,2)$. However, primary renal carcinoid tumor is a rare case and was firstly reported by Resnick in 1966 (1). In this case report, we aimed to focus on a case with welldifferentiated primary neuroendocrine carcinoma of the kidney pathologically diagnosed after partial nephrectomy in horseshoe kidney.

\section{Case report}

In a 37-year-old woman patient, examined due to incidentally diagnosed splenomegaly, a horseshoe kidney with a hypodense area $18 \times 12.5 \mathrm{~mm}$ in size at left kidney lower pole was observed by abdominal computed tomography $(\mathrm{CT})$ scans. Abdominal magnetic resonance imaging (MRI) confirmed a pathological area $18 \times 12.5$ $\mathrm{mm}$ in size at left kidney lower pole, in venous phase monitoring at T2 sequence with mild hypointense signal, with a mild suspicious contrast and exhibiting diffusion limitation in diffusional examination (Figure 1).

The patient was operated by left-open partial nephrecto-
Figure 1.

A. Horseshoe kidney formation and hypodense area $18 \times 12.5 \mathrm{~mm}$ in size at left kidney lower pole (red, lower arrow). Isthmus in the patient with horseshoe kidney anomaly (blue, upper arrow).

B. Monitoring at T2 sequence with mild hypointense signal of the area exhibiting diffusion limitation.

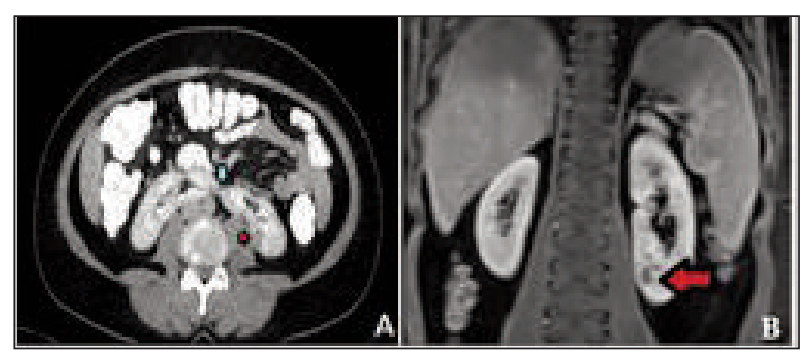

my zero ischemia with modified Chevron incision (Figure 2). No complication was observed during intraoperative and postoperative periods. At macroscopic pathological examination of the specimen, a $2 \mathrm{~cm}$ in diameter and cream color mass was observed, at microscopic examination, negative surgical borders, mitotic index 1/50 high-power fields (HPF) and no necrosis were observed. From immunohistochemical tests, CDX2 (-), CD10 (-), vimentin (-), CK7 (-),epithelial membrane antigen (EMA) (-), chromogranin focal (+), synaptophysin: common (+) and 8\% Ki-67 were observed. Microscopic and immunohistologic findings were in comply with primary neuroendocrine tumor of the kid-

\section{Figure 2.}

Exophytic mass lesion. Borders were determined by cautery at left kidney lower pole.

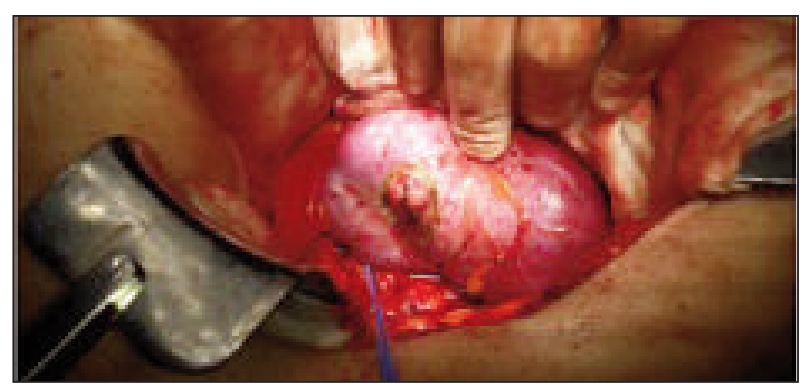

No conflict of interest declared. 
Figure 3.

A. Synaptophysin positive tumor cells.

B. Association of normal kidney tissue with tumor HEx4.

C. Neuroendocrine tumor HEx2O.

D. Neuroendocrine tumor HEx40.

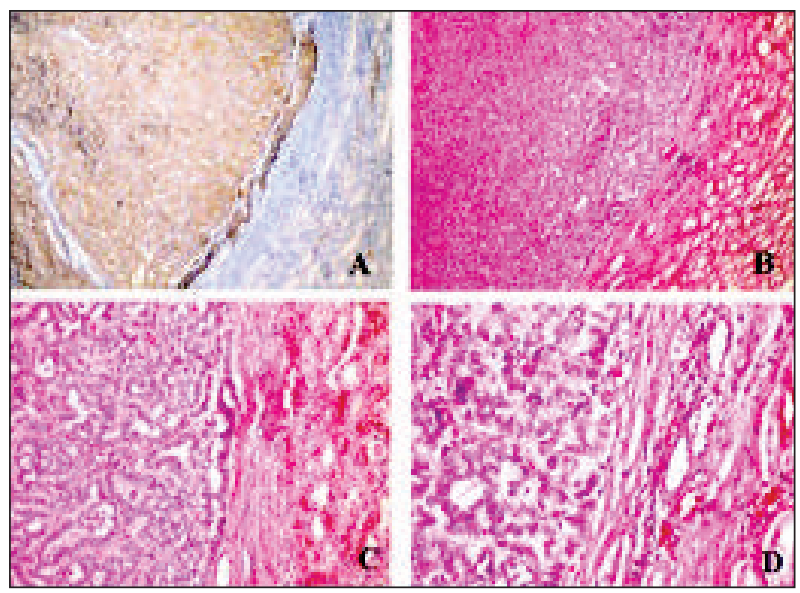

ney diagnosis (well-differentiated carcinoid tumor with trabecular and solid pattern) (Figure 3).

No pathology was observed at gastrointestinal system endoscopy, thorax computed tomography and 68GaDOTATATE positron emission tomography/computed tomography (PET/CT).

Neuroendocrine tumor markers were not found in urine and blood. The patient was followed by diagnosis of primary neuroendocrine carcinoma of the kidney and during 6 months follow-up period no local relapse or systemic metastasis was observed.

\section{Discussion}

Primary neuroendocrine carcinomas of the kidney are rare and their pathogenesis is still unknown as these tumors do not usually present in adult normal renal parenchyma, renal pelvis and ureter (2). Unlike renal cell carcinoma, renal carcinoid tumor is observed at an early age and in most cases the age at diagnosis is about 49 years and no gender dominance is present. Symptoms (e.g. carcinoid syndrome) are rare and it is incidentally diagnosed in 25-30\% of cases (2-4). They are clinically misdiagnosed with type 1 papillary renal cell carcinoma, mesonephric tumors, urothelial tumors, Wilms tumor and undifferentiated carcinoma (3).

Primary neuroendocrine carcinoma of the kidney do not have specific CT or MRI imaging. In general, primary renal neuroendocrine tumors show a mild contrast uptake as it's observed in radiological examinations of our case. Apart from this, it's characterized by cystic mass and sometimes by calcification at CT $(5,6)$.

The most dominant histological types of carcinoid tumors are trabecular or ribbon-like types. As a result of immunohistochemical studies, synaptophysin, chromogranin and CD56 showed positive and thyroid transcription factor (TTF-1), Wilms tumor protein (WT-1) and CDX2 showed negative $(2,7)$. Chromogranin, synaptophysin and CD56 positivities and CDX2 negativities of our case supported our neuroendocrine tumor diagnosis, and thus, we discarded renal cell carcinoma diagnosis. It's known that primary neuroendocrine carcinomas of the kidney are frequently presented in kidneys with anomaly. So far in studies in the literature, 100 patients have been diagnosed by similar cases and it has been observed that tumor development mostly occurs in horseshoe kidney. The incidence of neuroendocrine carcinoma in horseshoe kidney is 62 times higher than in normal kidneys (8). In their report examining clinical characteristics of 21 renal carcinoid tumors, Hansel et al. reported horseshoe kidney formation in 19\% of the patients (7).

Other kidney anomalies apart from horseshoe kidney are found as renal teratoma or teratoid malformation and polycystic kidney anomalies (1).

Our case of primary neuroendocrine tumor $2 \mathrm{~cm}$ in size, diagnosed in horseshoe kidney supports the literature. Primary neuroendocrine carcinomas of the kidney are known to stem from neuroendocrine cells and be lowgrade malignancy potential tumors. To determine prognostic state of these tumors, some indicators and clinical findings are proposed. Aung et al. reported about morphologic and molecular structures of 11 well-differentiated renal carcinoid tumors showing that distant metastasis were not present in tumors $5 \mathrm{~cm}$ in size or $\leq 2$ mitotic $10 \mathrm{HPF}$ (3). In an other review, Romero et al. reported that $50 \%$ of cases was metastatic and of which $45.6 \%$ was at the time first diagnosis.

They also stated that the risk of metastasis increases especially in patients over 40 years of age and with solid characterized and high mitotic activity tumors (2). Complete surgical resection is the primary treatment against primary neuroendocrine tumors of the kidney. Partial nephrectomy might be applied depending on the size of the tumor (9).

Octreotide or 68Ga-DOTATATE PET/CT is offered during follow-up as useful diagnostic tool in order to diagnose residual or hidden metastatic carcinoid tissue after surgical resection (10). Patients may develop local lymph node or distant organ metastasis after a long period of clinical course (7).

In our study, a local tumor $2 \mathrm{~cm}$ in size with low mitotic index was diagnosed and no distant metastasis was present. During follow-up after partial nephrectomy, no local or systemic relapse was observed.

\section{Conclusion}

Primary neuroendocrine carcinoma of the kidney are rarely observed and treated by curative treatment after complete surgical resection and show low-grade malignancy potential. Its presence in kidneys with anomalies should be kept in mind and, if there is not a clear definitive diagnosis of other kidney masses, it should be taken into consideration.

\section{REFERENCES}

1. Zhang Q, Ming J, Zhang S, Qiu X. Primary micro neuroendocrine tumor arising in a horseshoe kidney with cyst: report of a case and review of literature. Diagn Pathol. 2012; 7:126. 
2. Romero FR, Rais-Bahrami S, Permpongkosol S, et al. Primary carcinoid tumors of the kidney. J Urol. 2006; 176:2359-66.

3. Aung PP, Killian K, Poropatich CO, et al. Primary neuroendocrine tumors of the kidney: morphological and molecular alterations of an uncommon malignancy. Hum Pathol. 2013; 44:873-80.

4. Murali R, Kneale K, Lalak N, Delprado W. Carcinoid tumors of the urinary tract and prostate. Arch Pathol Lab Med. 2006; 130:1693-706.

5. Quinchon JF, Aubert S, Biserte J, et al. Primary atypical carcinoid of the kidney: a classification is needed. Pathology. 2003; 35:353-5.

6. Motta L, Candiano G, Pepe P, et al. Neuroendocrine tumor in a horseshoe kidney. Case report and updated follow-up of cases reported in the literature. Urol Int. 2004; 73:361-4.
7. Hansel DE, Epstein JI, Berbescu E, et al. Renal carcinoid tumor: a clinicopathologic study of 21 cases. Am J Surg Pathol. 2007; 31:1539-44.

8. Krishnan B, Truong LD, Saleh G, et al. Horseshoe kidney is associated with an increased relative risk of primary renal carcinoid tumor. J Urol. 1997; 157:2059-66.

9. Lane BR, Chery F, Jour G, et al. Renal neuroendocrine tumours: a clinicopathological study. BJU Int. 2007; 100:1030-5.

10. Gabriel M, Oberauer A, Dobrozemsky G, et al. 68Ga-DOTATyr3-octreotide PET for assessing response to somatostatin-receptormediated radionuclide therapy. J Nucl Med. 2009; 50:1427-34.

\section{Correspondence}

Kamil Gokhan Seker, MD

Emre Sam, MD

Selcuk Sahin, MD

Mustafa Gürkan Yenice, MD

Volkan Tugcu, MD

Abdulmuttalip Simsek, MD (Corresponding Author) simsek76@yahoo.com

Bakirkoy Dr.Sadi Konuk Education and Research Hospital

Department of Urology

Tevfik Saglam Caddesi No:11 Zuhuratbaba/Bakirkoy

Istanbul 34147, Turkey

Ayse Gul Aktas, MD

Bakirkoy Dr.Sadi Konuk Education and Research Hospital

Department of Pathology

Tevfik Saglam Caddesi No:11 Zuhuratbaba/Bakirkoy

Istanbul 34147, Turkey 